\title{
Does the number of categories perceived or the number rehearsed affect recall?
}

\author{
LAWRENCE C. PERLMUTER \\ Virginia Polytechnic Institute and State University, Blacksburg, Virginia 24061 \\ and \\ ROBERT KARSH and RICHARD A. MONTY \\ Behavioral Research Directorate, Human Engineering Laboratory \\ Aberdeen Proving Ground, Maryland 21005
}

\begin{abstract}
Subjects were required to tally mentally the frequency of occurrence of a set of letters sequentially presented. In one condition, four different letters were presented repeatedly in a haphazard sequence and were tallied into four groups or categories. In the second, or "combination condition," the identical four letters were tallied into only two categories. In the third condition, only two distinct letters were tallied into two categories. The poorest performance was observed with the use of four categories, while subjects in both two-category conditions performed nearly identically. The results were discussed in terms of imaginal processes involved in keeping track tasks and were compared with those found in memory organization tasks.
\end{abstract}

When subjects are required to remember the occurrence of a number of items whose presentation is uniquely represented in a set, they tend to recall these items in a sequence different from that in which they were presented (e.g., Mandler, 1967). The recalled items are generally reported in intrarelated groups or categories and subjects can recall reliably more items when categorization is permitted than when the subjects are required to recall the items in a manner which reflects the serial order of their presentation. This categorization effect has been repeatedly found in static-type tasks wherein items within the list are not repeated.

In a more dynamic task, intensively studied by Monty and his associates (e.g., Monty, Taub, \& Laughery, 1965), only a limited number of different items (typically two to four letters) are presented repeatedly in a haphazard sequence of up to 24 occurrences. The subject is required to mentally tally the frequency of occurrence of each item or letter. It has been found that very few subjects tally the number of letters by recalling their order of presentation. Rather, it appears that most subjects tend to picture a set of windows or bins, one corresponding to each of the letters to be tallied. Following presentation of each letter, an appropriate change is made in the corresponding category tally (Monty, Karsh, \& Taub,

Requests for reprints should be sent to Lawrence C. Perlmuter, Department of Psychology, Virginia Polytechnic Institute and State University, Blacksburg, Virginia 24061. The authors gratefully acknowledge the valuable assistance of Earl Pence and Karyn Griffith in collecting and analyzing the data. This paper may be reproduced in full or in part for any purpose of the United States Government.
1973), and at the end of the trial the subject reports the number of occurrences of each item or letter.

One of the factors affecting performance in this task is the number of different items to be kept track of or tallied. As the number of different letters increased from two to four, there was a corresponding increase in errors (Monty et al., 1965). This increment in errors presumably stems from interference with category rehearsal which apparently requires more time as the number of categories increases. In their study, however, the number of different letters and the number of different categories into which the different letters could be segregated were allowed to covary. Thus, it is unclear whether the resulting increase in errors was attributable to the increase in the number of different letters to be tallied or to the number of categories or bins into which these could be placed. It is possible to explicate this somewhat confounded relationship by holding one factor constant while manipulating the other factor.

This study then inquires what would result if subjects were presented with four different letters to be tallied in only two categories rather than four different letters to be tallied in four categories. Extrapolating from the investigations of Mandler (1967), it was predicted that the number of categories into which the letters must be tallied, rather than the number of different letters tallied, would most directly affect performance.

A second purpose, orthogonal to the first, relates to the particular letters to be tallied. There is some evidence that stimuli which can be more easily ordered (e.g., letters of the alphabet) are easier to keep track of than are stimuli such as geometric 
figures which are less easily ordered (Monty, 1968). Further, more recent evidence (Monty, Mazurczak, \& Perlmuter, Note 1) showed that adjacent letters of the alphabet (e.g., HIJK) are easier to keep track of than nonadjacent letters (e.g., CFIL). It was hypothesized that if letters from a common word such as DECK were utilized as categories, keeping track performance would be facilitated for the same reason that ordered stimuli (letters) lead to better performance than unordered symbols.

\section{METHOD}

\section{Subjects}

One-hundred fifty-three male and female students from Virginia Polytechnic and State University were given course credit for serving as subjects.

\section{Apparatus and Procedure}

The apparatus has been described more fully elsewhere (Monty et al., 1965). Briefly, the subjects were seated in front of an Industrial Electronic Engineers, Inc. rear projection display. Sequences of letters, seen as an outline of light against a dark background, were preprogrammed with Massey-Dickinson, Co. solid-state modules located in an adjacent room, and were projected one letter at a time onto the $3.8 \times 4.8 \mathrm{~cm}$ frosted glass surface. The subject's task was to observe and count mentally the number of occurrences of the letters presented and to record their tallies in writing at the end of each sequence or trial in the appropriately labeled columns.

The letter stimuli were drawn from either the familiar word "DECK," from a pronounceable nonsense syllable "DECL" or from the nonpronounceable nonsense syllable "DBCK." The subjects were randomly assigned to one of nine groups of 17 subjects each. Three groups were instructed to mentally keep a tally of the frequency with which letters drawn from one of the four categories (DECK, DECL, or DBCK) occurred. In the combination conditions, three groups were instructed to keep a combined mental tally of the four letters shown. That is, only two categories were used to keep track of four letters (DE-CK, DE-CL, or DB-CK). In addition, three groups were instructed to keep a mental tally of the frequency with which letters drawn from each of two categories (EC, DL, or BC) occurred. Note that the letter sequences and frequencies were identical for the groups keeping track of identical letters. That is, the group receiving DECK and the groups receiving DE-CK always were presented with an identical series on every trial. An identical relationship held between the groups keeping track of DECL and DE-CL as well as those keeping track of DBCK and DB-CK. Thus, the major difference between these three pairs of groups was in terms of the categorization requirements. In order to maintain comparability of the two letter groups with their respective four letter groups, the following procedure was used. In the EC condition, a Letter $\mathrm{E}$ was presented whenever the program presented D or E to the four-letter groups (DECK or $\mathrm{DE}-\mathrm{CK})$ and a $\mathrm{C}$ was presented whenever the program presented a $\mathrm{C}$ or a $\mathrm{K}$ to these four-letter groups. A similar procedure was followed in generating letter sequences in Groups DL and BC. Thus, on the average, the two-letter groups received twice as many presentations of each letter as did the four-letter groups. This was done in order to maintain equivalence of the total rumber of stimuli at each point throughout the experiment.

Trial length, defined as the total number of stimuli to be kept track of in a sequence, irrespective of their categories, was included as a within-subjects variable. Trial lengths of $6,12,18$, or 24 stimuli were presented haphazardly throughout the experiment. To permit assessment of practice effects, a total of 32 trials (sequences) were presented. Each of the four trial lengths was presented twice in random order during each of the first eight trials and twice more in different random orders during each of the remaining three blocks of eight trials. The number of times each letter was presented per trial was determined at random with the restriction that each letter occurred at least once on each trial. All stimuli were presented at the rate of one every second with a .5 -sec on-time and .5 -sec interstimulus off-time. A $2,500-\mathrm{Hz}$ tone signaled the beginning and end of each trial.

\section{RESULTS}

The sum of the absolute differences between the number of letters shown in each category and the number reported in each category was used as the measure of performance. For example, if five Ds, three Es, two Cs, and two Ks were shown on a given trial and the subject reported seven Ds, two Es, two Cs, and two Ks, then the errors would be two for the Ds and one for the Es for a total of three on that trial. For the groups whose categories represented either two letters or a combination of the two letters, the same scoring procedure was followed except that in the combination condition two letters presented were treated as one, i.e., five Ds + three Es = eight DEs. The absolute error scores for the two equal trial lengths within each block were summed and subjected to an analysis of variance (Butler, Kamlet, \& Monty, 1969) with "words" and conditions as between effects and trial length and blocks as within effects. The groups viewing two categories made an average of 1.52 errors per trial while the groups reducing four categories to two averaged 1.85 errors per trial and the groups rehearsing all four categories made an average of 3.29 errors per trial, resulting in a significant main effect for conditions $F(2.144)=65.70, p<.001$. The main effect for blocks, $F(3.432)=32.30, p<.001$, and trial length, $F(3.432)=554.52, p<.001$, both reached significance simply indicating that performance improved with practice or blocks and the longer the trial length the poorer the performance. while the Blocks by Trial Length interaction, $F(9.1296)=13.25, p<.001$, indicated that the longer the trial length the greater the improvement with practice. Of greater interest are the Blocks by Conditions interaction, $F(6,432)=2.18, p<.05$, and the Trial Length by Conditions interaction, $F(6.432)=21.07 . p<.001$, shown in Figures 1 and 2. respectively. The data underlying the first of these interactions indicated that as practice progressed, performance within each condition improved, but much more so for the subjects working with two categories and the combination condition than for the subjects rehearing all four categories. Three subsequent analyses pairing each of the levels of the conditions variable with each other revealed no interacttions of Conditions by Blocks. However, the main effects for the two-category groups vs. the four-category groups as well as the combination groups forming two categories vs. the four-category group both revealed significance at the .001 level of confidence, while there was no difference between the 
groups rehearsing only two categories. Finally, the data underlying the Trial Length by Conditions interaction simply indicated that while errors increased as a function of an increase in trial length for all conditions, they did so much more rapidly for the groups rehearsing all four categories. Subsequent analyses again revealed no differences between the two category groups and the combination groups forming two categories. Thus, the interaction is a function of the groups working with four categories interacting with each of the others at the .001 level of confidence. Words and all interactions with words failed to reach significance at the .05 level of confidence.

\section{DISCUSSION}

We have seen that when subjects are required to mentally keep track of the number of occurrences of four letters in two groups of two, performance is relatively better than when they must keep track of the letters in four distinct categories. This result confirms the hypothesis that subjects tally frequently occurring items by grouping them since the subjects in the fourcategory groups and the combinations groups each received the identical sequence and frequency of occurrence of the four letters. These data are unequivocal in demonstrating that the deterioration in performance which occurs when the number of different letters increase is attributable to the number of categories into which they are segregated rather than to the increase in the number of different items to be tallied. Secondly, these results extend the observation of Mandler (1967) and others who have shown that subjects can greatly expand the number of items which they can remember if categorization of like or similar items is permissible. Thus the present results extend the categorization effects from tasks in which individual items are presented on only a single occurrence to tasks in which a few items are repeatedly presented. Thirdly, since there is essentially no difference in performance between the groups receiving only two items and those keeping track of two pairs of items we can begin to understand something more about how subjects store information about frequency of occurrence, at least in the keeping track task. Howell (1973) has reviewed the problem of the representation of frequency in memory and in conformity with one of his proposals it can be concluded that subjects employ counting in tasks where actual frequency information is required.

Theoretically, it might be expected that performance in the combination conditions would be somewhat poorer than it is when only two categories are tallied as the former condition would seem to require an additional step or set of steps involved in entering a pair of letters into the appropriate bin or category. That is, when a single letter is represented by its corresponding category, the tallying process can be more directly approached than it can when either of two letters must initially be combined into a single category prior to the tallying process. Thus, this combination condition may require a perceptual or discriminal process which could serve as an additional source of error. However, the data are clear in showing that the combinational requirement does not disadvantage the subject's performance and that subjects can presumably enter either one or two different items into the same category with about the same degree of proficiency. This imaginal aspect of the task has recently been discussed by Monty et al. (Note 1). According to this notion, earlier discussed by Paivio (1971) and Monty et al. (1973) subjects perform this portion of the task with relative ease once they adopt the imaginal spatial strategy. Presumably, this additional step, at least with the particular items used in

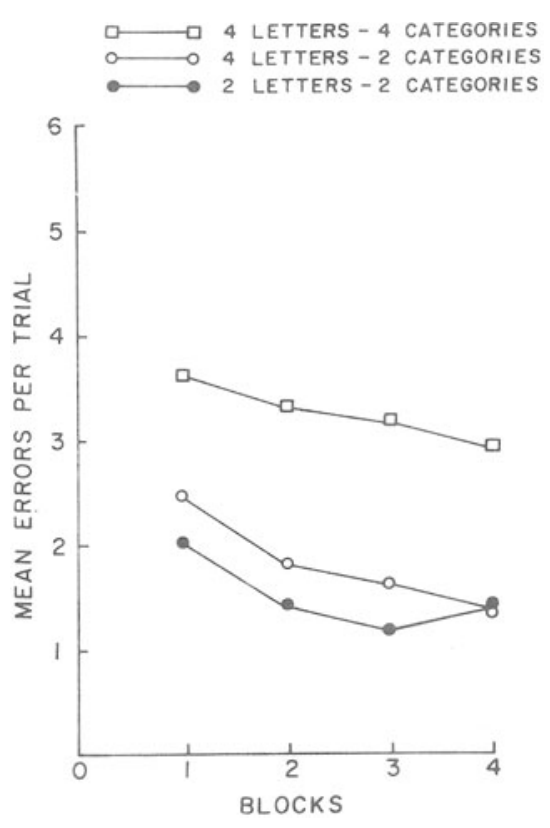

Figure 1. Mean number of errors per trial as a function of blocks and conditions.

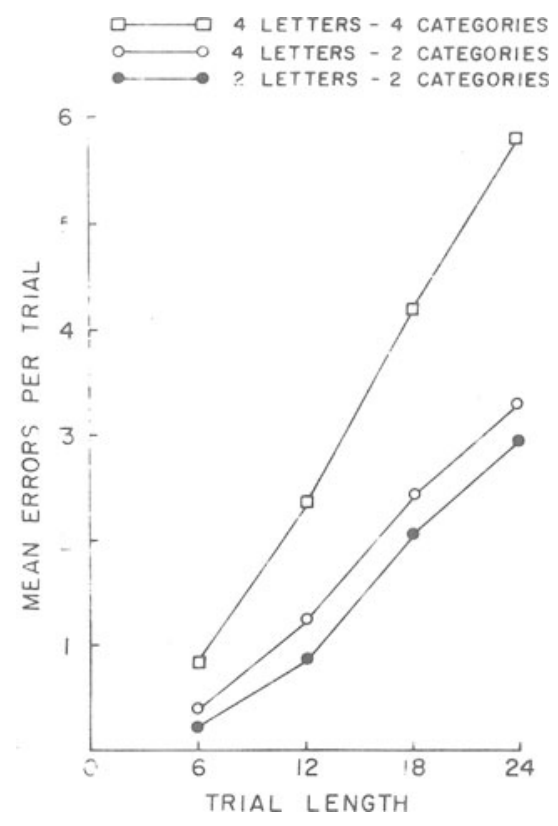

Figure 2. Mean number of errors per trial as a function of trial length and conditions.

this experiment contributes relatively little if any error to performance. Whether items which are not as readily ordered, such as geometric figures or tones would produce the combination or categorization effects as herein observed await further studies. It is, however, reasonable to expect this combinational effect to occur even when nonverbal stimuli are used since there is evidence from the free recall literature showing that subjects who are presented with pictorial stimuli (common 
objects) evidenced clustering by spatial array in their free recall data (Frost, 1971). Thus, in summary, the data seem to support the contention of Monty et al. (1965) that adequate rehearsal time is a major determinant of performance in this task, and that alterations in other aspects of this task show only minor influences on performance.

In this context, the significant Trial Length by Conditions interaction also warrants further attention. It will be recalled that this interaction revealed an increasingly large difference between the groups rehearsing two categories and the groups rehearsing four categories as a function of an increase in trial length. There are at least two possible explanations for this. First, the effect could stem from insufficient time to rehearse all four categories after each presentation of a new stimulus, thus as the trial length increases the number of errors should be cumulative (Monty et al., 1965). An alternative interpretation stems from differences in the relative rates of change of each category tallied. On the average, each category in the fourcategory condition had a rate of change of one-half the rate of change of the two-category conditions. As a result, it was necessary for subjects in the four-category condition to remember the current tallies in each window or bin twice as long, on the average, as subjects in the two-category groups. Further, as the number of letters per trial increased, the probability of the subject being required to remember one tally for a longer period of time is greater for the subject in the fourcategory group than for subjects in the two-category group. Thus these results point to an interesting counter-intuitive effect. Although the rate of presentation of stimuli for all subjects in all conditions is identical, the rate at which category totals are incremented in both groups keeping track of only two categories is relatively more massed temporally than it is for the group keeping track of four categories. Nevertheless, the two-category massed conditions are superior to the four-category distributed condition. Further, the interaction of Trials by Conditions is intriguing for another reason. That is, it is now clear that the effect of trial length is not influenced by the total value of any category since, on the average, total category value was twice as high in the groups with two categories as it was in the groups with four categories. For example, if 24 letters were presented on a trial, the average category value in the group keeping track of four distinct categories would approximate 6 while it would approximate 12 in the two-category group. Hence, it is apparent that the total value of the category is not a relevant variable in affecting performance at least with the present range of values.

Finally, the experiment failed to show any effect whatsoever of the manipulation of letter sets as defined by the differential ease of pronounciability of these sets. There are several possible reasons for this null effect. The most parsimonious explanation is that for subjects presented with sets of four letters, three of these four letters were alphabetically adjacent and Monty et al. (Note 1) have shown that letter adjacency aids performance in this task. In the case where subjects received only two letters, some of which were and some of which were not adjacent alphabetically, the task may be so easy that letter adjacency does not influence performance. Again, the lack of a pronounciability effect is not surprising if one assumes that categories are spatially arrayed images and that rehearsal is restricted to category tallies rather than to category names and tallies, at least when ordered and familiar materials are used. Thus, the imaginal aspects of this task may be spatially tagged rather than verbally named. This latter proposal is consonant with some recent work of Peterson (1975) who showed that an imaginal strategy aids importantly in memory.

\section{REFERENCE NOTE}

1. Monty, R. A., Mazurczak, J., \& Perlmuter, L. C. The effect of inherent onder on imagery, spatial organization and remembering. Submitted for publication, 1976.

\section{REFERENCES}

Butler, D. H., Kamlet, A. S., \& Monty, R. A. A multipurpose analysis of variance FORTRAN IV computer program. Psychonomic Monograph Supplements, 1969, 2. 301-319 (Whole No. 32).

Frost. N. Clustering by visual shape in the free recall of pictorial stimuli. Journal of Experimental Psychology. 1971. 88. 409-413.

Howell, W. C. Representation of frequency in memory. Psychological Bulletin, 1973, 80, 44-53.

MANDler. G. Organization in memory. In K. W. Spence \& J. T. Spence (Eds.). The psychology of learning (Vol. I). New York: Academic Press. 1967.

Monty, R. A. Spatial encoding of strategies in sequential shortterm memory. Journal of Experimental Psychology. 1968. 77. 506-508.

Monty, R. A., KaRsh. R., \& TAUB, H. A. Imagery and interference in sequential short-term memory. Canadian Journal of Psychology, 1973, 27, 220-226.

Monty, R. A.. Taub, H. A.. \& Laughery, K. A. Keeping track of sequential events: Effects of rate. categories and trial length. Journal of Experimental Psychology. 1965. 69. 224-229.

Paivio. A. Imagery and verbal processes. New York: Holt, Rinehart and Winston. 1971.

Peterson. M. J. The retention of imagined and seen spatial matrices. Cognitive Psychology. 1975. 7. 181-193.

(Received for publication December 3. 1975.) 